\title{
A Neural Solution for the Level 2 Trigger in Gamma Ray Astronomy
}

\author{
S. Khatchadourian*, J.-C. Prévotet and L. Kessal \\ Laboratoire ETIS - CNRS UMR 8051 - ENSEA - Université de Cergy-Pontoise, France \\ E-mail: khatchadourian@ensea.fr, prevotet@ensea.fr, kessal@ensea.fr
}

The HESS project is a major international experiment currently performed in gamma astronomy. This project relies on a system of four Cherenkov telescopes enabling the observation of cosmic gamma rays. The outstanding performance obtained so far in the HESS experiment has lead the research labs involved in this project to improve the existing system: an additionnal telescope is currently being built and will soon take place within the previous telescope system. This telescope is designed to be more sensitive to the detection of low energy particles than the others, leading to an increase of the number of collected particle images. In this context which is tightly constrained in terms of latency, physicists have been compelled to design an additionnal L2 trigger in order to deal with a huge amount of data. This trigger aims at selecting images of interest (ie. gamma particles) and rejecting all other events that are associated to noise. Contrary to classical methods that consist of strong cuts based on Hillas parameters, we propose an original approach based on artificial neural networks. In this approach, collected events are first handled by an intelligent preprocessing level whose purpose consists in applying images transformation algorithms. In a second step, a basic neural network ensures the classification of events within three classes (gamma, muon and proton). Several types of preprocessing and their associated neural classifier will be considered and compared in this presentation in terms of recognition capabilities.

XI International Workshop on Advanced Computing and Analysis Techniques in Physics Research April 23-27 2007

Amsterdam, the Netherlands

\footnotetext{
* Speaker.
} 


\section{Introduction}

Neural networks [1] have successfully been implemented in a wide variety of applications and have demonstrated their efficiency compared to other classical techniques in pattern recognition and classification. One interesting field in which neural techniques have been of a great interest is the triggering of particles in physics experiments [2]. The triggering approach consists in taking a decision according to a specific event. Due to the promising results obtained by neural networks in this context, it has seemed interesting to explore this specific solution in the HESS (High Energy Stereoscopic System) experiment [3].

The HESS experiment is based on the atmospheric Cherenkov technique: as a high energy cosmic ray hits the atmosphere, it creates an extensive air shower by interaction with the atmosphere. This phenomenon is known as Cherenkov light. The HESS experiment consists in studying this light in order to detect gamma-rays. This experiment, based in Namibia, has been collected data since 2003 and keeps on delivering highly interesting results and demonstrating that the field has entered a new era. The current HESS system (phase 1) is composed of four $12 \mathrm{~m}$ diameter imaging Cherenkov telescopes, arranged on a square of $120 \mathrm{~m}$ side. Each telescope features a $107 \mathrm{~m}^{2}$ mirror area and contains 960 photomultipliers tubes (PMTs). By combining the information of, at least two telescopes, at the same time, the system makes intensive use of the stereoscopic approach. The HESS system is designed for the detection of particles of high energy $(>100 \mathrm{GeV})$.

According to the huge amount of data to be collected, a trigger system composed of two levels is integrated in the HESS experiment: a level 1 (L1) and a central trigger. Such a trigger system is useful to keep the relevant events on-line in order to exploit them off-line. The L1 consists in removing isolated pixels within an image by applying basic thresholds. These pixels are typically due to noise. The central trigger allows to perform time correlation between all the telescopes: interesting data of each telescope are sent to the central trigger. If there are relevant data from at least two telescopes at the same time, the event is stored for future off-line image analysis and source reconstruction.

After more than a year of observation about twenty sources have been detected with an unequalled significance and an angular resolution that allows a fine study of extended source morphology.

\section{Second phase of the HESS project}

The outstanding performance obtained so far in the HESS experiment has led the research labs involved in this project to improve the current sensitivity of the existing system [4]: a Very Large Cherenkov telescope (VLCT) of $24 \mathrm{~m}$ diameter is currently being built and will take place in the center of the previous telescope system. The VLCT will reduce the energy threshold in stereoscopic mode allowing to collect more photons for a shower at a given energy. It will function in a monoscopic mode for lower energies. Moreover, the VLCT camera will also be improved: 2048 PMTs instead of the 960 PMTsof the previous telescopes. All these improvements will contribute to a better sensitivity of the HESS system. The quality and the reconstruction of the gamma parameters will thus be improved. The HESS2 experiment will upgrade the HESS1 experiment by:

- adding a new HESS2 event class (energies from $10 \mathrm{GeV}$ to $50 \mathrm{GeV}$ will now be collected), 
- increasing the sensitivity of the existing telescope system for energies from $50 \mathrm{GeV}$ to $100 \mathrm{GeV}$,

- improving the resolution for energies upper than $100 \mathrm{GeV}$.

Moreover, in order to increase the sensitivity and thus obtain the best performances, the PMT trigger threshold will be reduced. In this context, the quantity of data to be collected by the VCLT will drastically increase. A simulated trigger rate of $2.5 \mathrm{kHz}$ is expected, reaching up to $20 \mathrm{kHz}$ for the trigger worst conditions. The trigger rate associated with the huge amount of data to be processed on line ( $240 \mathrm{GBauds}$ in approximatively $10 \mu \mathrm{s}$ ) has led the collaboration to propose a new efficient trigger scheme composed of three levels: two trigger levels (L1 and L2) and a central trigger.

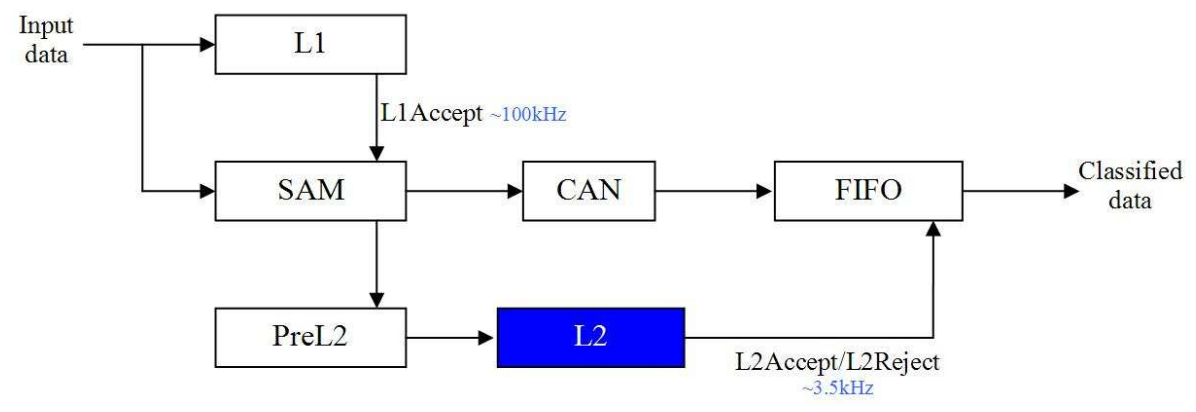

Figure 1: Trigger system in HESS-II

The figure 1 describes the function of the trigger system. Data coming from the camera are stored in an analog memory (SAM) allowing the storage of the entire image. In parallel, data are also sent to the level 1 trigger (L1) which as the same structure as in HESS-I. This level aims at taking a decision regarding the nature of the event. It notably generates a binary signal indicating whether a specific event has to be kept (L1Accept) or rejected (L1Reject). If the L1Accept signal is valid, the complete image is stored and converted to a serie of digital data. These data are stored in a FIFO until a new L2Accept/L2Reject signal coming from the level 2 trigger (L2) decides to keep or reject this event. In parallel data are sent to the PreL2 step which thresholds the images at three energy levels. The image is then sent to the L2. The L1 and L2 decisions are respectively and approximately expected at a $100 \mathrm{KHz}$ and $5 \mathrm{KHz}$ rate.

\section{A pattern recognition issue}

In the HESS2 experiment, the observations to study consist in the signatures of hadronic or electromagnetic showers on the telescope. Three types of particles have to be discriminated : gammas, muons and protons. In this context, the interesting particle type is gamma and muons or protons may be considered as observation noise. The nature of images differs according to the incident energies and to the impact distance. Examples of particle's signatures are depicted in figure 2. Images to be processed by the L 2 are provided by a PreL2 level and are composed of 2048 pixels, each of these coding in three different energy levels. 

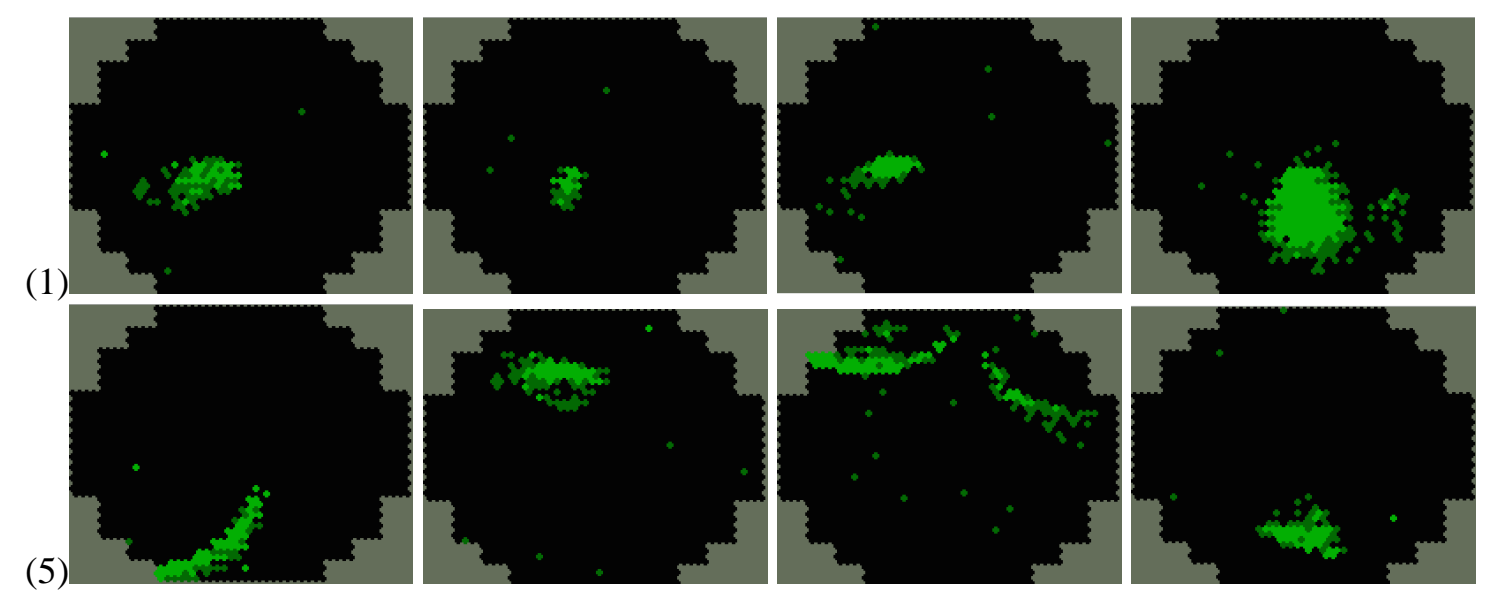

Figure 2: Gamma (1-4), muon (5-6) and proton (7-8) images of different energies.

\subsection{Classical approach}

This pattern recognition issue is often treated by efficient algorithms that aim at extracting geometrical information from the particles. Astrophysicists generally perform simple thresholds on the images according to physical characteristics in order to isolate interesting events. They usually compute Hillas parameters [5] and apply cuts on the obtained results. The idea of this algorithm is to adjust a bi-dimensional ellipse on the image according to the particle signature (see figure 3 ). Parameters such as the center of gravity (CoG), length, width, surface, area and the $\alpha$ angle are computed and presumably contain all the useful information. According to the parameters values, the particles are classified in the three different classes : gammas, muons and protons.

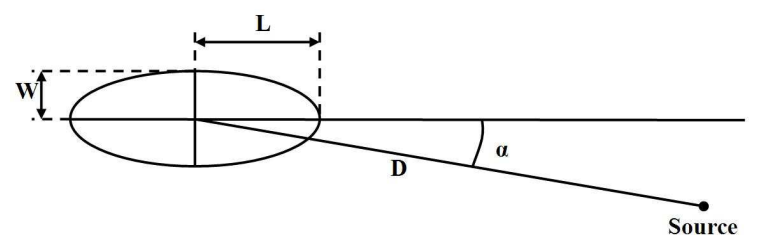

Figure 3: Overview of Hillas parameters

Since Hillas parameters constitute an efficient way of discriminating between particles, it has seemed appropriate to extend theses concepts by considering non-linear systems that are also known as effective classifier and that have shown rather impressive results in quite similar problems. A new trigger system based on a neural network architecture has then been envisaged.

\subsection{Neural solution}

The proposed neural L2-trigger scheme is detailed in figure 4. It is composed of two main blocks: the neural classifier and its associated preprocessing that are described in the next sections. 


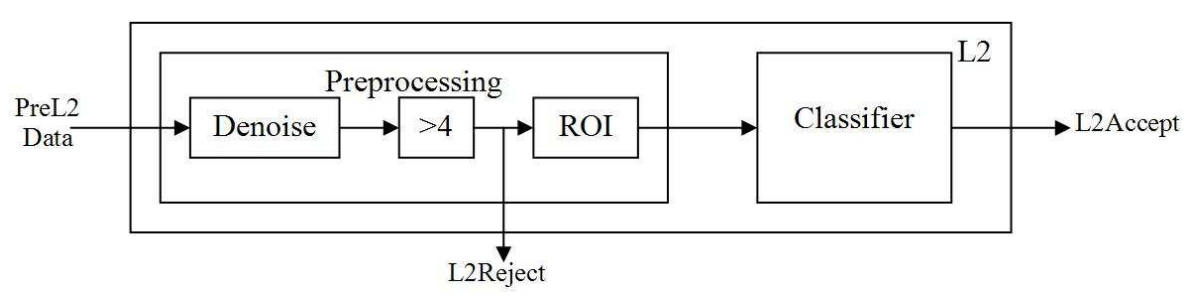

Figure 4: Level 2 trigger system

\subsubsection{Preprocessing}

In the neural network context, preprocessing is a major and inescapable step. Its role consists in applying more or less complex transformations on input data in order to help the network in its classification task. The main role of preprocessing is generally to reduce the dimensionality of the problem by extracting significant information from the input data and thus reduce the size of the neural network. Moreover, the learning process is also drastically simplified since the number of examples to furnish (which is exponential according to the number of parameters) is also reduced. Nevertheless, one the most significant drawback of such preprocessing relies in its relative complexity and it is often necessary to perform an efficient compromise between the complexity of the neural network and its associated preprocessing.

The envisaged preprocessing is divided into three steps:

- Removing isolated pixels: In the L2 trigger context, images coming from the camera may contain several isolated pixels, typically due to noise. These pixels have to be removed in order to obtain the most representative image of the particles. A prefiltering algorithm consists in applying a mask on all pixels of the image and keeps relevant information.

- Rejecting data that may not be relevant for the classifier : Some of the biggest clusters in the images are smaller than 4 pixels. Since a classifier can not make a decision with such a low information, these clusters are automatically rejected.

- Extracting the most interesting features of the image: This third step consists in extracting a region of interest (RoI). A statistical study on the available data set has shown that $99 \%$ of the particles may be contained in a window of $21 * 21$ pixels. The biggest cluster of the image is located in the image and inscribed in this window after a centering step. Nethertheless a problem persists with circular images that cannot be inscribed in this area. This issue has basically been circumvented by taking into consideration only partial parts of the whole image (typically arcs of a circle).

\subsubsection{Neural network}

The structure of the neural network is a simple multi-layer perceptron (MLP) with 441 inputs corresponding to the pixels of the RoI presented before, 1 hidden layer and 3 outputs corresponding to the three types of particles to identify (gammas, muons and protons). Each output neuron features a TanH activation function that ranges between -1 (rejected) and 1(accepted). Since outputs are 
continuous, only the maximum of the three values is retained and corresponds to the particle to be identified.

The selected image patterns are respectively composed of 2000 gammas, 2000 muons and 2000 protons in the learning base and the remaining data are distributed between the testing and the validation base. The events of the learning base are randomly chosen on the entire data set.

\subsection{Results}

A comparison between the presented approaches are described in table 3.3. Results are expressed in terms of gammas recognition rate and muons or protons rejection rate.

The applied thresholds for the Hillas approach follows this procedure:

- if $\mathrm{CoG}<\mathrm{t}$ then the event is recognized as a gamma particle.

- if $\mathrm{CoG}>=\mathrm{t}$ and $\alpha<20 \mathrm{deg}$ then the event is recognized as a gamma particle,

- otherwise the event is rejected.

$\mathrm{t}$ is a parameter adjusted according to the data set. This parameter has been chosen in order to accept the maximum of gamma particles while rejecting the maximum number of muons and protons. It has been fixed to 0.5 .

\begin{tabular}{|l|c|c|c|}
\hline & Gamma & Muon & Protn \\
\hline Neural solution & $80 \%$ & $75 \%$ & $75 \%$ \\
\hline Hillas filter & $60 \%$ & $56 \%$ & $37 \%$ \\
\hline
\end{tabular}

The obtained results show that the neural solution performs a better classification than the Hillas algorithm. $80 \%$ of the gamma particles are correctly classified. The rejection rate on muons and protons particles is also significantly better with the neural solution. These results are encouraging and demonstrate that the neural approach constitutes a successful alternative to classical methods as a L2 trigger.

\section{Conclusion}

The classical Hillas approach is usually utilized by the astrophysicists as a trigger solution. Unfortunately, the cuts performed on the results often reject too much gamma particles. The neural approach, which has already shown efficient results in other physics experiment as [2], constitutes an interesting solution. Moreover, this type of algorithms may be easily implemented in real-time on hardware as shown in [6]. This seems particularly interesting since the processing time in the HESS 2 experiment is tightly constrained : Only $10 \mu$ s are available to make a decision at L2. In this context, it is envisaged to explore other preprocessing solutions that may take into account the intrinsic nature of the collected images. One field of investigation could consist in exploiting the geometrical properties of the particles in order to help the classifier in performing its task. 


\section{References}

[1] C.M. Bishop, Neural networks for pattern recognition, Oxford University Press, 1996.

[2] C. Kiesling The H1 Neural Network Trigger Project, Proc. Advanced Computing and Analysis Techniques in Physics Research, In P. Bhat et M. Kasemann, editors, American Institute of Physics, 2001.

[3] W. Hofmann, The H.E.S.S. Project, Proc. Symposium The Universe Viewed in Gamma-Rays, Eds. R. Enomoto, M. Mori, S. Yanagita, Univ. Academy Press, p. 357, 2002.

[4] P. Vincent, H.E.S.S. collaboration, H.E.S.S. Phase II, Proc. 29 Int. Cosmic Ray Conference, Pune, India, Vol. 5, 163-166

[5] A.M. Hillas, Proc. 19th ICRC, La Jolla 3, 1985.

[6] J-.C. Prévotet et al., Moving NN Triggers to Level-1 at LHC Rates, Nuclear Instruments and Methods in Physics Research A, 2003, apr, 502, 511-512. 